\section{The creation of functioning gastrojejunostomy following}

3 Wei Qiao ${ }^{1,2 *}$, Yi Lv², Zhaoqing $\mathrm{Du}^{1,2}, \mathrm{Hui} \mathrm{Li}^{1}$, Lixue $\mathrm{Du}^{1}$, Haitian $\mathrm{Hu}^{1}$, Dichen $\mathrm{Li}^{3}$

$4{ }^{1}$ Department of Hepatobiliary Surgery, Shaanxi Provincial People's Hospital, Xi'an, Shaanxi

5 Province, China

$6 \quad \quad 2$ National Local Joint Engineering Research Center for Precision Surgery \& Regenerative

7 Medicine, Shaanxi Provincial Center for Regenerative Medicine and Surgical Engineering, First

8 Affiliated Hospital of Xi'an Jiaotong University. Xi'an, Shaanxi Province, China

$9 \quad{ }^{3}$ State key laboratory for manufacturing systems engineering, Rapid manufacturing research

10 center of Shaanxi Province, Xi'an Jiaotong University, Xi'an, Shaanxi Province, China

11 * Corresponding author: Department of Hepatobiliary Surgery, Shaanxi Provincial People's

12 Hospital. No.256 Youyi West Road, Xi'an, Shaanxi Province, 710068, China. Tel/fax: +86

13 02985251331(2640). E-mail addresses: qiaowei030816@126.com 


\section{Abstract:}

Background: Magnetic compression for creating gastrojejunostomy has many advantages according to previous studies. However, following mechanical device release after healing, the anastomotic stenosis becomes the pivotal point. Methods: Rectangle-shaped magnets were used for magnetic compression in rabbits. Both paclitaxel-loaded magnets and a strategy of pyloric ligation were chosen to improve the gastrojejunostomy. Based on these choices, the half-capsule was applied to occlude the pylorus after anastomotic formation. The size and patency of the anastomoses were analyzed to evaluate the efficacy of these approaches. A histological examination was also performed. Results: The positive effect of ligating the pylorus on gastrojejunostomy was significantly greater than that achieved using paclitaxel-loaded magnets during either short- or long-term follow-up. There were fewer scar tissue and collagen fibers at the anastomotic site in the treatment group than in the control group. The anastomotic aperture was of great interest at 9 months after the ligation of the pylorus following magnetic compression. In the view of the jejunum, although the aperture was barely visible, gastric juice was continuously spilling through it like a spring, and the aperture was clearly visible from the stomach side. All half-capsules failed to block the pylorus. Conclusion: The effect of paclitaxel on maintaining gastrojejunostomy patency was temporary. The ligation of the pylorus ensured the long-term patency of 
36 gastrojejunostomy, and the aperture was comparable to the pylorus which

37 could play an anti-reflux role. Further studies for the sort of gastrointestinal

38 aperture are being planned.

39 Keywords: magnetic compression anastomosis, gastrojejunostomy,

40 anastomotic stenosis, pyloric ligation, paclitaxel-loaded 


\section{Introduction}

Magnetic compression anastomosis (MCA) is a promising mechanical method for digestive reconstruction. In 1978, Kanshin and colleagues[1] reported that steady compression by magnets could be utilized to establish sutureless gastro-duodenal and ceco-jejunal anastomosis and provided apparent advantages over traditional manual sutures. Over the past 40 years, this technique has been showed to have a large number of advantages, including its convenience in use, time saving, less invasive nature, and cost-effectiveness. In addition to the speed and certainty with which an anastomosis can be made, MCA can also provide progressive compression, thus contributing to necrosis of the interposed tissues by increasing strength beyond other mechanical anastomoses[2], such as Murphy's button and the Valtrac Ring, which have represented a breakthrough in the area of anastomotic procedures in the more than 100 years since $1892[3$, 4].

The magnets maintain a permanent pressure at the anastomotic point until their release and improve the performance of circumferential airtightness so that anastomotic fistulas and bleeding can be avoided in the early stage. Following mechanical device release after healing, however, the contraction and stenosis of the stoma are the pivotal points, upon which the utility of the devices rests[5-8]. In the prior study published from our 
group, anastomotic contraction occurred immediately after the removal of compression magnets, regardless of whether rectangle- or cylinder-shaped magnets were used[9]. The character of contraction recovers the rhythm of dietary excretion after the reconstruction of the alimentary tract and, to some extent, can be effectively utilized to prevent digestive reflux. However, anastomotic stenosis may result in considerable morbidity or even mortality. During this process, the proliferation of scar tissue plays a critical role.

The present study investigated a solution for stenosis of the gastroenteric stoma after magnetic compression in rabbits. Base on the aforementioned research, the following issues were explored: (1) the effect of the closure of the initial outflow tract on maintaining functioning gastroenteric stoma, (2) the relationship of the primary size of the anastomosis is to its permanent patency, and (3) whether local paclitaxelloaded magnets can suppress the production of collagen fibers in the early stage to maintain long-term patency.

\section{Methods}

The committee on the Ethics of Animal Experiments at Xi'an Jiaotong University approved the protocol (No. XJTULAC2017-1010). The animals used in our experiment received humane treatment in strict accordance with the Animal Care and Use Committee of Xi'an Jiaotong University. 
83 All surgeries were performed under sodium pentobarbital anesthesia, and

84 an effort was made to minimize suffering.

85

\section{Experimental animals}

Japanese big ear white rabbits ( $\mathrm{n}=28,50 \%$ male) weighing 2.5 to 3.5

$\mathrm{kg}$, were obtained from the Laboratory Animal Center of Xi'an Jiaotong University and housed under controlled conditions, including a 12-h lightdark cycle and a temperature of $20 \pm 2^{\circ} \mathrm{C}$ with free access to foot and water. Before the experiment, the rabbits were adaptively fed for one week.

The animals were randomly divided into 5 groups: the initial diameter of the anastomoses was measured after MCA $(n=4)$; MCA was performed using paclitaxel-loaded magnets (MCA-PTX, $\mathrm{n}=8$ ); MCA was followed by the ligation of the pylorus (MCA-LP, $\mathrm{n}=8$ ); MCA was followed by a sham operation (control group, $\mathrm{n}=5$ ); and the magnet device for compression was connected to a half-capsule (MCA-HC, $\mathrm{n}=3$ ).

\section{Design of the magnets}

Rectangle-shaped magnets were used for magnetic compression to establish gastroenteric anastomosis with shells of polylactide. Their length, width and height were $16 \mathrm{~mm}, 5 \mathrm{~mm}$ and $2.5 \mathrm{~mm}$, respectively. The magnets were magnetized perpendicular to their stress surface.

In the present study, an MCA device incorporated a pair of magnets 
103 called the gastric magnet and jejunal magnet according to their location. In 104 the MCA-PTX group, the magnet device was wrapped in paclitaxel-loaded 105 poly(lactic-co-glycolic acid), approximately $9 \mathrm{mg}$ paclitaxel per device. In 106 the MCA-HC group, the jejunal magnet was connected to a silk suture that

was $25 \mathrm{~cm}$ in length. The other end of the line was tethered to a polythene tube device through a pore in a half-capsule, which was composed of polylactic acid and was $10 \mathrm{~mm}$ in length and $8 \mathrm{~mm}$ in diameter. The line was twined around the tube device and eventually encased in the halfcapsule when it was introduced into the stomach. According to the design, when the MCA device fell off the anastomotic site, the half-capsule would block the pylorus due to the traction of the connecting line.

\section{General procedure}

Anesthesia administered as intravenous pentobarbital $(30 \mathrm{mg} / \mathrm{kg})$ was followed by fixation on the operating table in the supine position with a straight neck. In the present experiment, the magnets and half-capsules were sequentially introduced into the stomach along a ferromagnetic guidewire, as described in the previous experiment[9]. Then, the jejunal magnet was squeezed into the jejunum through the pylorus. Once at the intended position, the jejunal magnet combined with the gastric magnet in the body of the stomach. This operation complied with surgical sterile standards. After the rabbits recovered from anesthesia, they were returned 
124 to their cages and allowed free access to food and water.

125

126

127

128

129

130

131

132

133

134

135 141 were used in SPSS 22 for statistical analysis. $P<0.05$ was considered to 142 indicate statistical significance. The sex of the animals was not considered

143 a factor in this study.

An abdominal X-ray was taken immediately to mark the initial

position of the magnet device as the base level, and this data was used to confirm whether the device became unexpectedly separated or fell off the anastomotic stoma during the experimental period.

\section{Specimen collection}

When the endpoint was achieved in the experiment, the animals were euthanized with an excess of pentobarbital. The anastomosis was immediately harvested, and its diameter was measured in the natural state. Finally, both hematoxylin-eosin and Masson's trichrome staining were performed to observe histomorphology by optical microscopy.

\section{Statistical analysis}

The diameter of the anastomotic stoma was measured in millimeters $(\mathrm{mm})$ and was presented as the average of the long and short diameter. The force per square centimeter exerted at the magnet device surface was shown as the associated pressure. The quantitative data were expressed as the mean \pm standard deviation. Student's $t$-test and one-way ANOVA 


\section{Results}

In this study, all animals survived to the endpoint. There was no

146 intestinal obstruction, fistula, or infection in any of the groups. Each

147 incision healed well. When the magnet pairs fell off, they were freely

148 extruded from the anus. The formation time of the anastomotic stoma was

$14912.1 \pm 1.4$ days following the MCA procedure $(\mathrm{n}=20)$ and $11.4 \pm 0.9$ days

150 after the procedure when paclitaxel-loaded magnets were used $(\mathrm{n}=8, P>$ $1510.05)$.

In the test of MCA-HC, all three half-capsules failed to act as expected to block the pylorus, and they were extruded from the pylorus before the magnet devices released (Fig 1). These rabbits were randomly transferred into the group of measuring initial diameter, the control group, or the MCA-LP group, respectively.

\section{Fig 1. Abdominal X-ray examination at 2 weeks after magnetic} at the site of gastrojejunostomy. The dotted oval indicates the contour of the stomach.

163 jejunal loop with the gastrojejunostomy significantly increased in each

164 group. The initial gastrointestinal anastomosis was $5.0 \pm 0.5 \mathrm{~mm}$ in 
165 diameter (Fig 2A), and the calculated area was $19.6 \mathrm{~mm}^{2}$, which was not 166 in accordance with the expected value of $80 \mathrm{~mm}^{2}$ given a relative 167 contraction of $75.5 \%$.

168 The anastomotic diameter was reduced to $2.2 \pm 0.2 \mathrm{~mm}$ at 50 days after 169 the MCA procedure $(P<0.01, v s$. the initial diameter; Fig 2B). In the 170 present study, a diameter of $7.1 \pm 0.2 \mathrm{~mm}$ was measured at 50 days after 171 the operation in the MCA-PTX group $(P<0.01$ vs. control group; Fig 2C), 172 while the diameter was $8.5 \pm 0.4 \mathrm{~mm}$ in the MCA-LP group $(P<0.01 v s$. 173 control group; $P<0.01$ vs. MCA-PTX group; Fig 2D).

Fig 2. Gross appearance of gastrojejunostomy. (A) The initial 175 anastomosis. The anastomoses at 50 days after establishment (B, in control) using magnets followed by the sham operation, (C) using paclitaxel-loaded

177 178 179 180 181 182 magnets and (D) using magnets followed by the ligation of the pylorus. The diameter of the anastomotic stoma was $5.0 \pm 0.3 \mathrm{~mm}$ at 9 months after MCA-LP $(P>0.05$ vs. the initial diameter; $P<0.01$ vs. the diameter at 50 days after MCA-LP; Fig 3). The aperture of anastomosis was of great interest at 9 months after the procedure in the MCA-LP group. On the outside view, the side-to-side anastomoses between the stomach and jejunum were complete (Fig 3A). In a window sectioning the jejunum, although the aperture was barely visible, gastric juice was continuously spilling through it like a mountain spring (Fig 3B), and the aperture was clearly visible from the stomach side (Fig 3C). The gastrojejunostomy was 
187

188

189

covered by mucosa with fibrosis in the submucosal and muscular layers. There was less scar tissue and fewer collagen fibers at the anastomotic site in the experimental group than in the control group (Fig 4).

Fig 3. The aperture of the anastomosis at 9 months after the procedure in the MCA-LP group. (A) Outside view showing the side-to-side anastomosis between the stomach and jejunum. (B) Section through the jejunum showing that the aperture was barely visible, but the gastric juice was continuously spilling through it like a mountain spring. (C) The aperture from the stomach side. The black arrow points to the anastomotic site.

Fig 4. Histological sections of gastrojejunostomy. (A, E) The initial anastomosis. The anastomoses at 50 days after establishment (B, F) using magnets followed by the sham operation, $(C, G)$ using paclitaxel-loaded magnets and $(\mathrm{D}, \mathrm{H})$ using magnets followed by the ligation of the pylorus. (Top: hematoxylin-eosin staining. Bottom: Masson staining)

Nevertheless, the bypass established between the stomach and jejunum was closed at 9 months after the procedure in the MCA-PTX group and was not covered by mucosa. The results of both gross examination and histological sections were shown in Fig 5.

\section{Fig 5. The closed gastrojejunostomy at 9 months after the procedure} in the MCA-PTX group. (A) Gross appearance. Histological examination stained with hematoxylin-eosin (B) and Masson staining (C). 
The magnets firmly attracted to each other with no accidental

210 separation. The compression force applied by the paired magnets was 9.1

$211 \pm 0.4 \mathrm{~N}$, and the associated pressure was $11.4 \pm 0.5 \mathrm{~N} / \mathrm{cm}^{2}$ with no spacing.

212 Considering the thickness of the interposed tissue, the force was $4.4 \pm 0.9$

$213 \mathrm{~N}$ with an associated pressure of $5.6 \pm 1.0 \mathrm{~N} / \mathrm{cm}^{2}$ according to the distance 214 of $1 \mathrm{~mm}$.

\section{Discussion}

It is generally believed that both fibroblast proliferation and the synthesis of collagen fiber play important roles in the process of anastomosis establishment. Nevertheless, excessive collagen accumulation

219 causes pathological scar formation during wound healing and acts as a 220 critical factor leading to an anastomotic stricture. Paclitaxel has been 221 shown to have the anti-fibrotic effect[10]. In the MCA-PTX group, 222 although the effect of paclitaxel on the synthesis of collagen fiber was 223 negative, it neither postponed the formation of gastrojejunostomy nor 224 resulted in the anastomotic leakage. These results indicated that the 225 experimental group showed apparent advantages on gross and histologic examination. Compared with the control group, the experimental group had 227 less scar tissue, which has been shown to be helpful in reducing the 228 incidence of anastomotic stenosis[11, 12]. 
230

231

232

beneficial until creating a stenosis. In traditional approaches, the scar gradually forms during the inflammation phase, approximately one month after the operation[13]. In this study, the first observation date was set at the 50 days after the operation. In the control group, the aperture had contracted to less than one-half of its original diameter. In the previous study that used smaller magnets $(7.2 \times 3.5 \times 2.0 \mathrm{~mm})$, the initial diameter of gastrojejunostomy was $1.5 \mathrm{~mm}$, and the gastrojejunostomy was completely closed within 12 days (Qiao W, unpublished data). The primary size of the anastomosis is related to its permanent patency.

The local administration of drugs such as paclitaxel-loaded magnets, can improve the patency of the anastomotic stoma in the early stage, but its long-term effect was not substantial. The positive effect of ligating the original outflow tract-pylorus on re-established gastrojejunostomy was significantly greater than the local effect of paclitaxel during either short(at 50 days) or long-term (at 9 months) follow-up. After the establishment of gastrojejunostomy by magnetic compression, the timely closure of the original tract can avoid anastomotic stricture.

The type of gastrointestinal aperture observed at 9 months after the procedure in the MCA-LP group was comparable to the pylorus which could play an anti-reflux role. This finding is interesting enough that surgeons should re-evaluate the advantages of magnetic compression anastomosis. The limitation of this part of the study was the relatively small 
252

253

254

255

271 which could play an anti-reflux role. Based on this study, further studies

272 for the sort of gastrointestinal aperture are being planned.

sample size.

was the minimum reported in all studies of successful gastrojejunostomy[13, 14]. This investigation may facilitate a factor of robustness in the design of future devices. It should be noted that the pressure between mating magnets varies with the thickness of the interposed tissue, which evolves over time during the process of magnetic compression.

In the MCA-HC group, all three half-capsules passed through the pylorus before the magnet devices fell off the anastomoses, and no halfcapsule was found in the stomach. These findings indicate that the halfcapsules used in this trial were not suitable for the occlusion of the pylorus, but the novel strategy for occluding the pylorus after anastomotic formation is still worth further investigation.

\section{Conclusions}

The magnets with larger sizes are recommended to establish a stoma in the digestive tract. The effect of paclitaxel is temporary to maintain the gastrointestinal patency. The ligation of the pylorus ensures the long-term patency of gastrojejunostomy, and its aperture is comparable to the pylorus 


\section{References}

274 1. Kanshin NN, Permiakov NK, Dzhalagoniia RA, et al. Sutureless anastomoses in gastrointestinal

275 surgery with and without steady magnetic field (experimental study). Arkh Patol 1978;40:56-61.

276 2. $\mathrm{Xu} \mathrm{ZF,} \mathrm{Li} \mathrm{ZJ,} \mathrm{Sun} \mathrm{YL,} \mathrm{et} \mathrm{al.} \mathrm{A} \mathrm{novel} \mathrm{spherical} \mathrm{magnetic} \mathrm{compression} \mathrm{device} \mathrm{for} \mathrm{colorectal}$

277 anastomosis in a Swine model. J Laparoendosc Adv Surg Tech A 2015;25:323-329.

278 3. Dietz UA, Debus ES, Hirt AL, et al. From Murphy's button to the Valtrac Ring. 100 years in search

279 of a paradigm. Zentralbl Chir 1999;124:653-656.

280 4. Mokros W. The indications for the Valtrac-ring--Report on 1015 Anastomoses. Zentralbl Chir $281 \quad 2001 ; 126: 1005-1008$.

282 5. Keen WW. Case of Cancer of the Hepatic Flexure of the Colon Producing Intestinal Obstruction;

283 Temporary Relief by an Artificial Anus; Later Re-establishment of the Continuity of the Bowel by Ileo-

284 colostomy by Means of Murphy's Button. Ann Surg 1893;17:652-666.

285 6. Lallemant Y. Esophageal stenosis after Murphy's button (utilized in treatment of hemorrhages of 286 esophageal varices. J Fr Otorhinolaryngol Audiophonol Chir Maxillofac 1978;27:51-54. anastomosis ring (BAR). Rozhl Chir 2000;79:429-432.

289 8. Lakyova L, Toporcer T, Bober J, et al. A rare complication following anastomosis suturing using a 290 biofragmentable Valtrac anastomosis ring--a case review and literature overview. Rozhl Chir $291 \quad 2010 ; 89: 446-449$.

292 9. Qiao W, Shi A, Ma F, et al. Further Development of Magnetic Compression for Gastrojejunostomy 


\section{Supporting information}




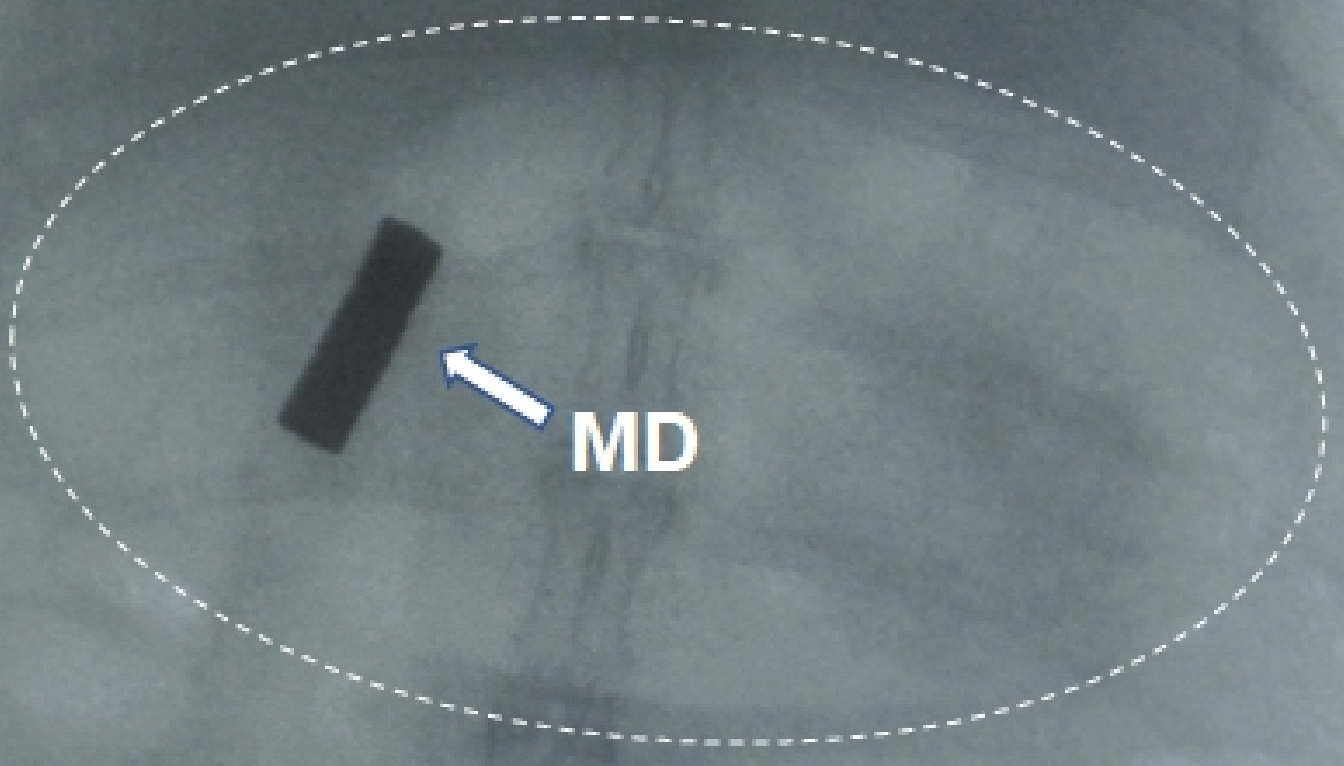

- $\mathrm{HC}$

Figure 


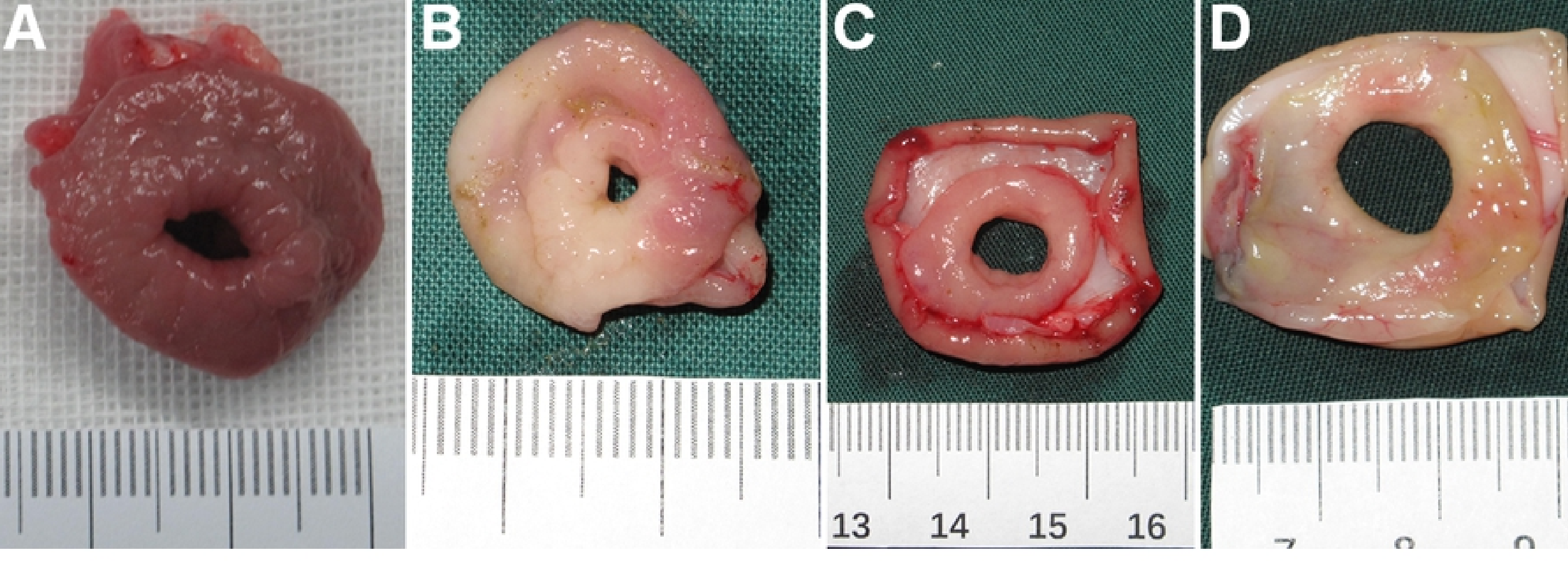

Figure 


\section{A \\ A}

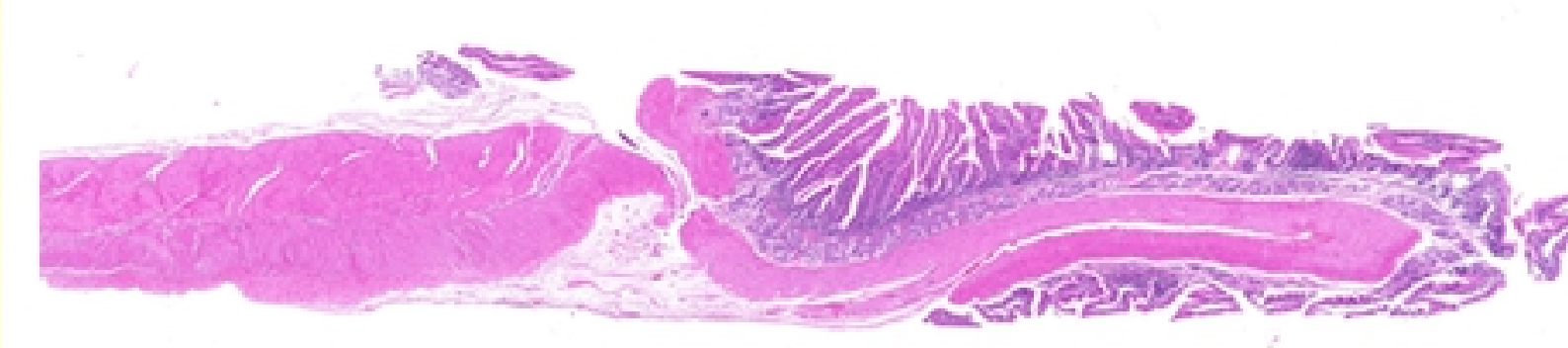

C

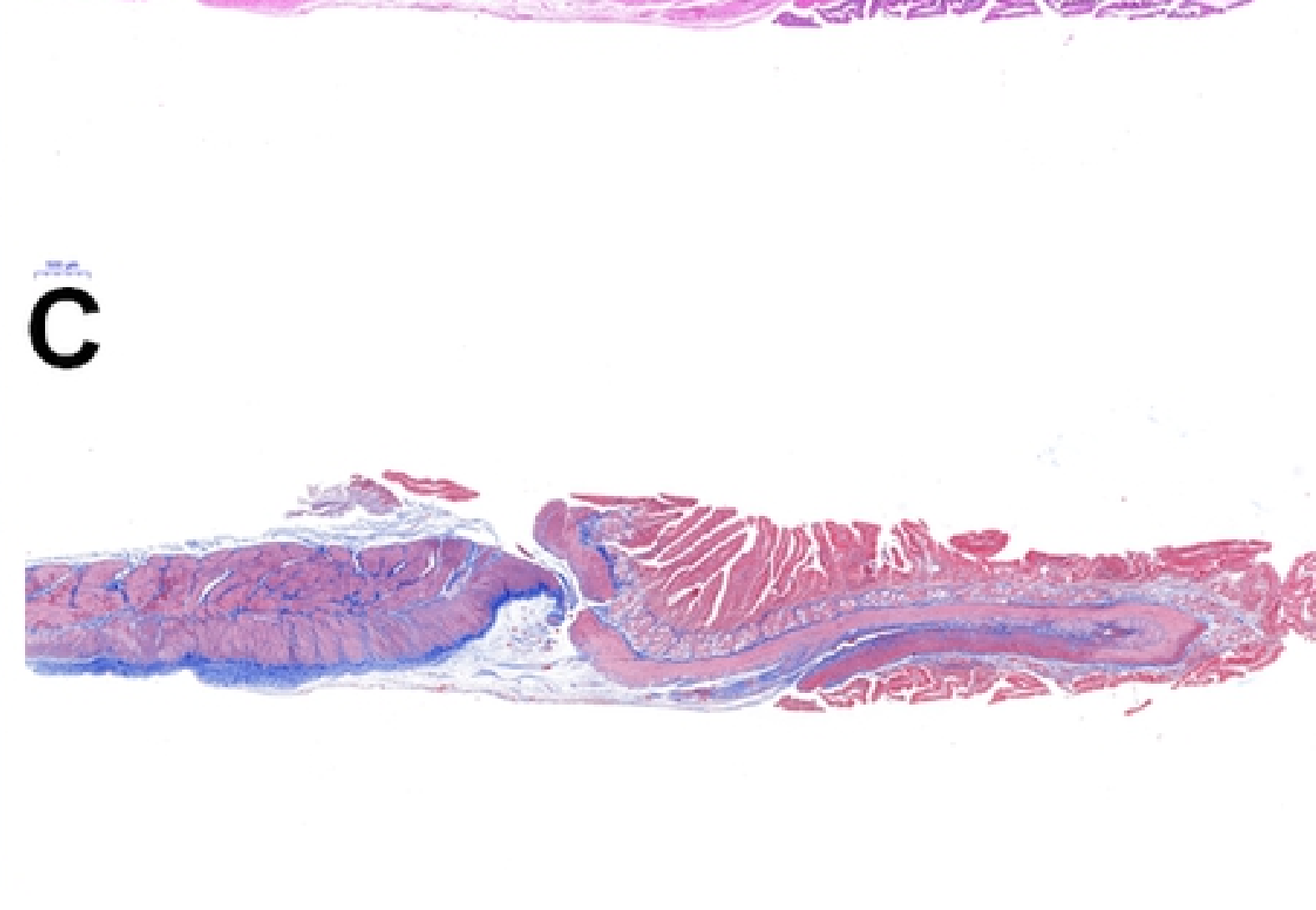

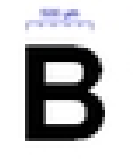 \\ B}

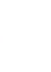
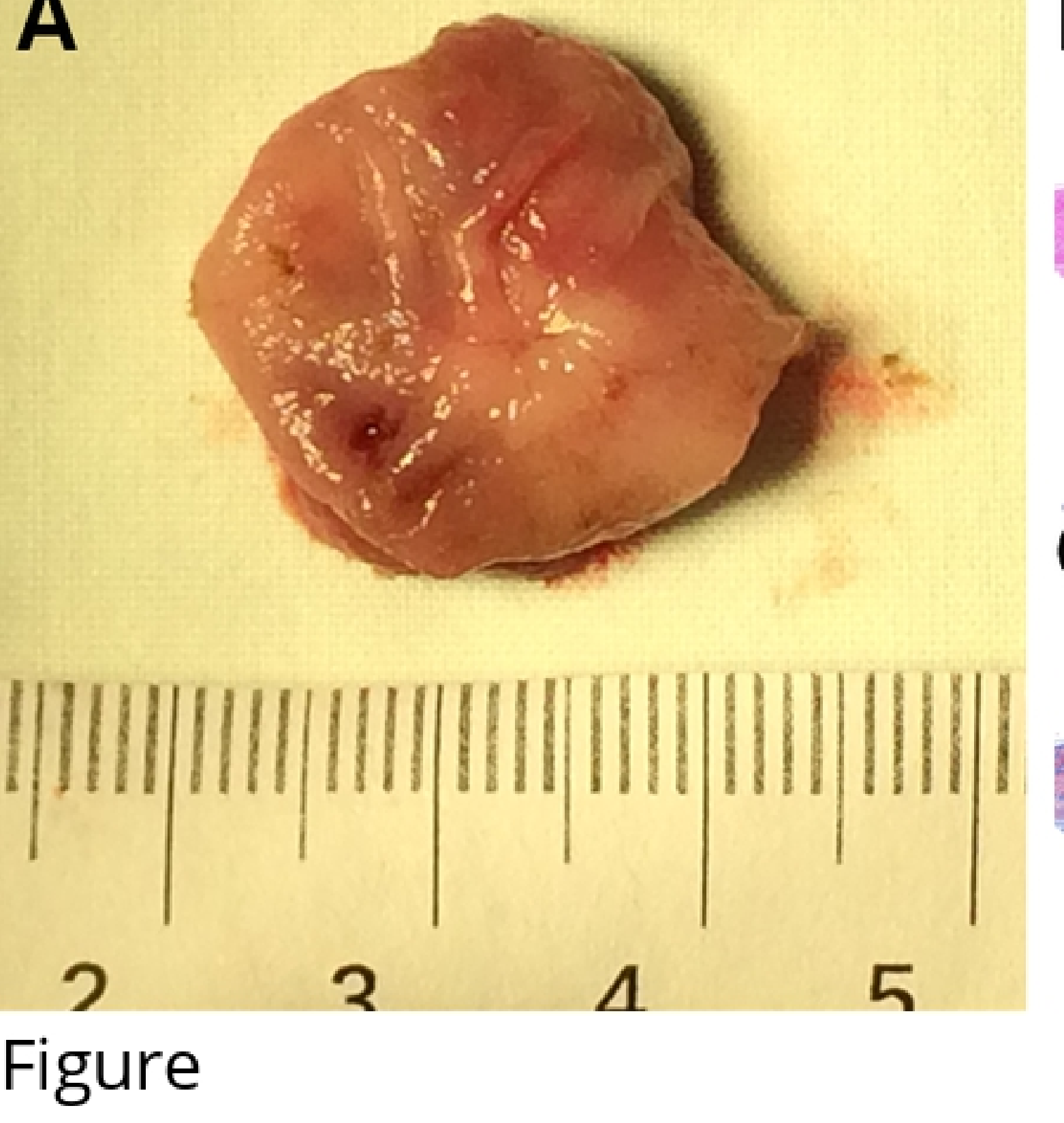

Figure

III
2

4

n

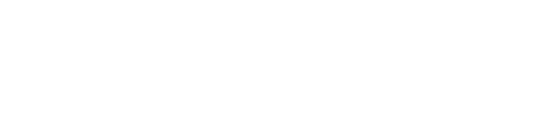

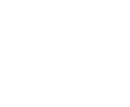

